\title{
Measurements of heavy flavour production in association with $W$ and $Z$ bosons with the ATLAS detector
}

\section{Ben COOPER* on behalf of the ATLAS Collaboration}

University College London, UK

E-mail: b.cooper@ucl.ac.uk

\begin{abstract}
The most recent measurements of heavy flavour production in association with $W$ and $Z$ bosons with the ATLAS detector at the LHC are presented: a measurement of $Z+b$-jet production using $36 \mathrm{pb}^{-1}$, a measurement of $W+\geq 1 \mathrm{~b}$-jet production using $4.6 \mathrm{fb}^{-1}$, and a measurement of $W$ production in association with a charm hadron using $4.6 \mathrm{fb}^{-1}$ of collision data. The analyses themselves are briefly described, and the main results presented, with particular emphasis on the comparisons with NLO predictions.
\end{abstract}

The European Physical Society Conference on High Energy Physics

18-24 July 2013

Stockholm, Sweden

\footnotetext{
* Speaker.
} 


\section{Introduction}

Owing to it's experimentally more challenging nature the production of heavy flavour ( $b$ or $c$ quarks) in association with vector bosons ( $\mathrm{VB}+\mathrm{HF}$ ) is a process that is relatively unexplored at hadron collider experiments in comparison to the (flavour inclusive) light quark dominated measurements of $W$ plus jets and $Z$ plus jets production [1, 2, 3]. It is however a process for which an improved understanding is becoming increasingly important at the LHC, since it forms a major background to several new physics searches [4], and in particular to the search for $H \rightarrow b \bar{b}$ production in the $\mathrm{W} / \mathrm{Z}$ associated mode $[5,6]$. In the following we briefly describe the recent measurements of VB+HF by the ATLAS [7] collaboration at the LHC. The large datasets that have been accumulated at the LHC in the 2011 running have enabled some of the first differential crosssection measurements of $\mathrm{VB}+\mathrm{HF}$ to be made, greatly improving our understanding of how well the latest next-to-leading-order (NLO) predictions reproduce the kinematics of these events. In addition, by examining the production of a single heavy flavour quark in the final state, as opposed to pair-production, one can obtain an improved understanding of how best to treat heavy flavour in the initial state, and of the heavy flavour content of the proton parton density function (PDF).

\section{2. $\mathbf{Z}+b$-jet cross-section}

ATLAS has produced a fully unfolded particle-level measurement of the inclusive (per jet) cross-section for $b$-jet production in association with a $Z$ boson [8], using $36 \mathrm{pb}^{-1}$ of $\sqrt{s}=7 \mathrm{TeV}$ LHC $p$ - $p$ collision data collected in 2010. The cross-section measurement is carried out in a fiducial region which closely relates to the selection at detector-level. Events containing $Z$ bosons are identified via the electron or muon decay channels, requiring $p_{T}^{\text {lepton }}>20 \mathrm{GeV},\left|\eta^{\text {lepton }}\right|<2.5$ and $76<m_{l l}<106 \mathrm{GeV}$. Jets are clustered using the anti $-\mathrm{k}_{\mathrm{T}}$ algorithm [9] with radius $\mathrm{R}=0.4$, and candidate $b$-jets with $p_{T}^{\text {jet }}>25 \mathrm{GeV}$ and $\left|\eta^{\text {jet }}\right|<2.1$ identified by displaced vertex tagging. The $b$ jet yield is extracted from the vertex mass distribution of the candidate $b$-jets. At the particle-level, a jet is identified as a $b$-jet if there is a stable b-hadron with $p_{T}>5 \mathrm{GeV}$ within $\Delta R<0.3$ of the jet, a definition that is applied consistently across all ATLAS analyses involving $b$-jets.

The measured cross-section of $3.55_{-0.74}^{+0.82}$ (stat) ${ }_{-0.55}^{+0.73}$ (syst) \pm 0.12 (lumi) pb is compared to an MCFM [10] parton-level prediction that is corrected for non-perturbative effects and final state radiation from the Z-decay leptons. The MCFM prediction is $3.88 \pm 0.58 \mathrm{pb}$, in good agreement with the data within the theoretical and experimental uncertainties.

In addition a further complimentary measurement is presented, where the $b$-jet yield in $Z$ events is divided by the inclusive yield of $Z$ events in the fiducial region to give a measurement of the average number of $b$-jets per $Z$ event, thus effectively normalising by the inclusive $Z$ production cross-section. The measured number of $\left(7.6_{-1.6}^{+1.8}(\mathrm{stat})_{-1.2}^{+1.5}(\mathrm{syst})\right) \times 10^{-3}$ is consistent with the prediction of MCFM $\left((8.8 \pm 1.1) \times 10^{-3}\right)$, and also of the predictions of ALPGEN [11] + Herwig [12] and SHERPA [13] leading-order generators.

\section{3. $\mathbf{W}+\geq \mathbf{1} b$-jet cross-section}

ATLAS has produced a fully unfolded particle-level measurement of the cross-section for the production of a $W$ boson in association with at least one $b$-jet, in exclusive 1 jet, 2 jet and $1+2$ jet 


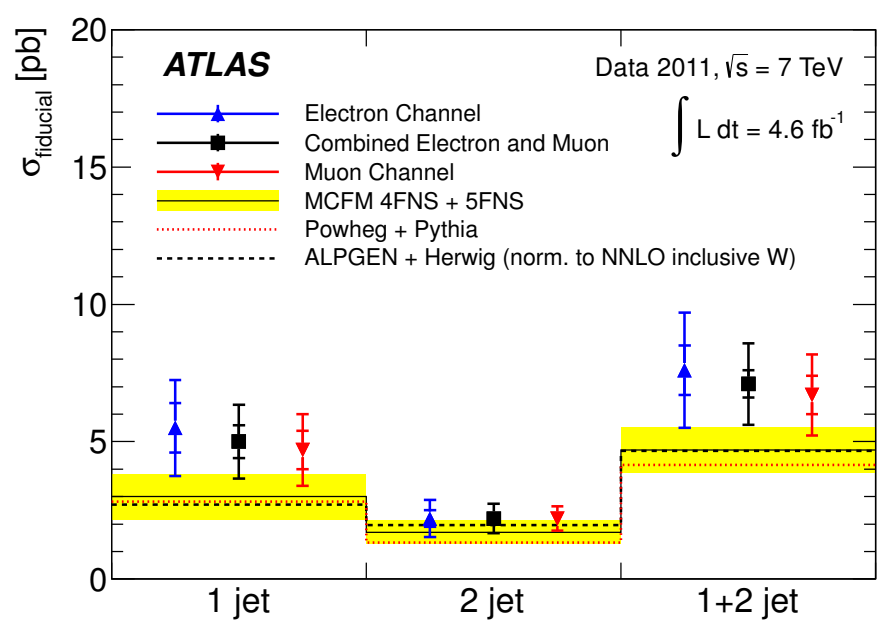

Figure 1: The measured $W+\geq 1 b$-jet cross-section in each of the 1 jet, 2 jet and $1+2$ jet bins, compared to the predictions of MCFM, POWHEG + Pythia and ALPGEN + Herwig [14]. The theoretical uncertainties on the MCFM prediction are displayed as the yellow band, and are dominated by the scale uncertainty and the uncertainty in the DPI correction. The MCFM prediction includes both five-flavour number scheme (5FNS) and four-flavour number scheme (4FNS) processes i.e. processes with and without a b-quark in the initial state.

bins [14]. The full dataset of $4.6 \mathrm{fb}^{-1}$ of $\sqrt{s}=7 \mathrm{TeV}$ LHC $p$ - $p$ collision data collected in 2011 is used. Events containing a $W$ boson are identified by requiring an isolated electron or muon candidate that satisfies stringent quality criteria, in combination with large missing $E_{T}$. The jets in these events are clustered with the anti- $\mathrm{k}_{\mathrm{T}}$ algorithm with radius $\mathrm{R}=0.4$, and jets with $p_{T}^{\text {jet }}>25$ $\mathrm{GeV}$ and $\left|\eta^{\text {jet }}\right|<2.1$ considered as candidate $b$-jets if they are "b-tagged" using a neural net based b-tagging algorithm [15]. The event selection requires exactly one b-tagged jet, in order to control

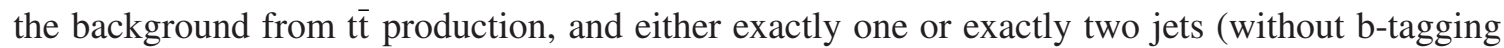
requirements).

The yield of $W+b$-jet events is determined by fitting the neural net b-tag weight distribution for the candidate $b$-jets, separately in the 1 and 2 jet bins and as a function of $b$-jet $p_{T}$. In this fit templates for the $W+b$-jets signal and $W+c$-jets and $W+$ light jets backgrounds are all taken from MC generators, with floating normalisation. Templates for the large $t \bar{t}$ and single top backgrounds are taken from $\mathrm{MC}$, with normalisation constrained using data control regions.

In Figure 1 is shown the measured fiducial cross-section in each of the 1 jet, 2 jet and $1+2$ jet bins, compared to the predictions of MCFM, POWHEG [16] + Pythia [17] and ALPGEN + Herwig. The ALPGEN prediction is scaled by a NNLO inclusive $W$ normalization factor. The MCFM prediction is at the parton-level, but with corrections applied for non-perturbative effects (fragmentation and the underlying event) and double-parton interactions (DPI). The uncertainties on the MCFM prediction are dominated by the scale uncertainty and the uncertainty in the DPI correction. Although there is apparently some tension, the measurement and MCFM NLO prediction are consistent within uncertainties. 


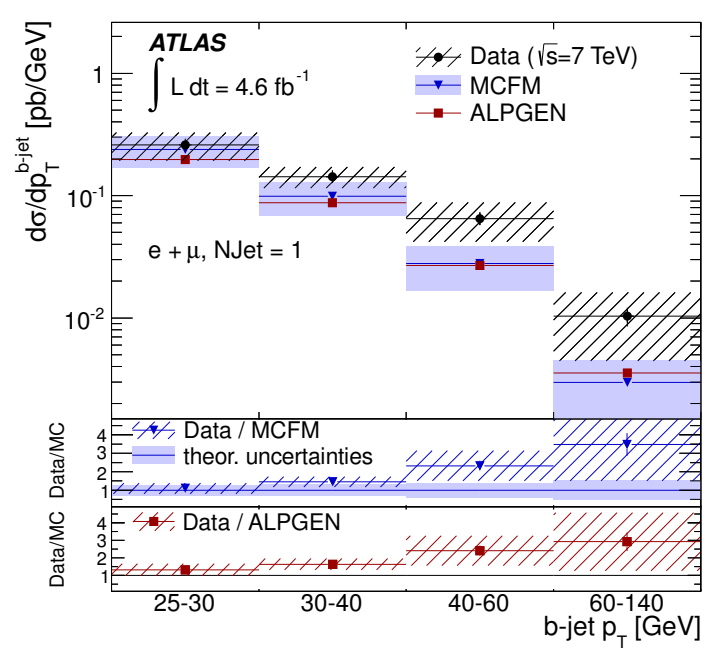

(a)

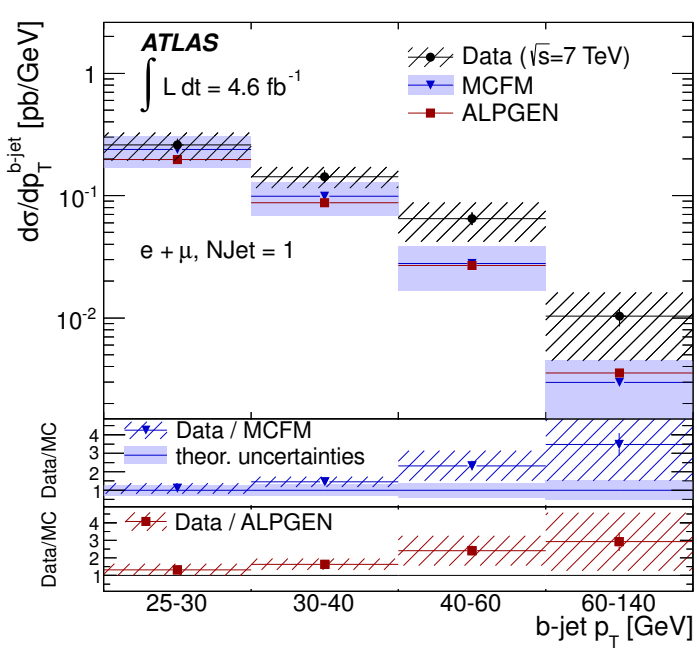

(b)

Figure 2: The differential $W+\geq 1 b$-jet cross-section as a function of the leading $b$-jet $p_{T}$ in the 1 jet (a) and 2 jet (b) bins [14].

The large statistics of the 2011 dataset also allowed, for the first time in VB + HF studies, a differential measurement of the cross-section for $W+\geq 1 b$-jet production as a function of $b$-jet $p_{T}$. This is shown in Figure 2, separately for the 1 jet and 2 jet bins. The data is compared to the corrected MCFM prediction, and the particle-level ALPGEN + Herwig prediction. One can see that, although the measurement and predictions are in agreement within uncertainties, there is evidence of a systematic mis-modelling of the shape by the theory, especially when one considers that the data uncertainties are strongly correlated between bins.

\section{4. $\mathrm{W}+$ charm hadron cross-section}

ATLAS has produced a fully unfolded particle-level measurement of the the production of a $W$ boson in association with a charm hadron [18], reconstructed in the decay modes $D^{+} \rightarrow K^{-} \pi^{+} \pi^{+}$ and $D^{*+} \rightarrow D^{0} \pi^{+}$with $D^{0} \rightarrow K^{-} \pi^{+}, D^{0} \rightarrow K^{-} \pi^{+} \pi^{0}$ or $D^{0} \rightarrow K^{-} \pi^{+} \pi^{-} \pi^{+}$, and their charge conjugate states. The full dataset of $4.6 \mathrm{fb}^{-1}$ of $\sqrt{s}=7 \mathrm{TeV}$ LHC $p$ - $p$ collision data collected in 2011 is used. The cross-section for $W^{ \pm}+D^{(*) \mp}$, where the $W$ boson and charm hadron have opposite charge, is measured in a fiducial region where the same sign contribution is subtracted (OS-SS). This subtraction enables the $W+c$ final state to be isolated from $W+c \bar{c}$, and hence gives sensitivity to the strange quark content of the proton PDF. Events containing a $W$ boson are identified by requiring an isolated electron or muon candidate and large missing $E_{T}$, and $W^{ \pm}+$ $D^{(*) \mp}$ events selected by explicitly reconstructing the $D^{(*) \mp}$ decays in the ATLAS inner detector [7]. The OS-SS yield is determined through a fit to the invariant mass after same sign subtraction of the $K \pi \pi$ system for $D^{ \pm}$, or similarly the mass difference $\Delta \mathrm{m}=\mathrm{m}\left(\mathrm{D}^{*}\right)-\mathrm{m}\left(\mathrm{D}^{0}\right)$ after same sign subtraction for the $D^{(*) \pm}$,

Figure 3 shows the measured $W^{ \pm}+D^{* \mp}$ and $W^{ \pm}+D^{\mp}$ OS-SS cross-sections, compared to six aMC@NLO [19] NLO predictions which each use a different PDF that vary in their treatment of 


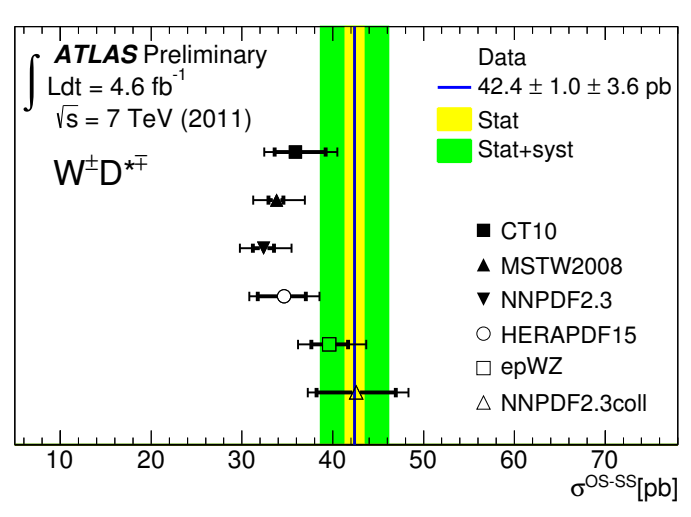

(a)

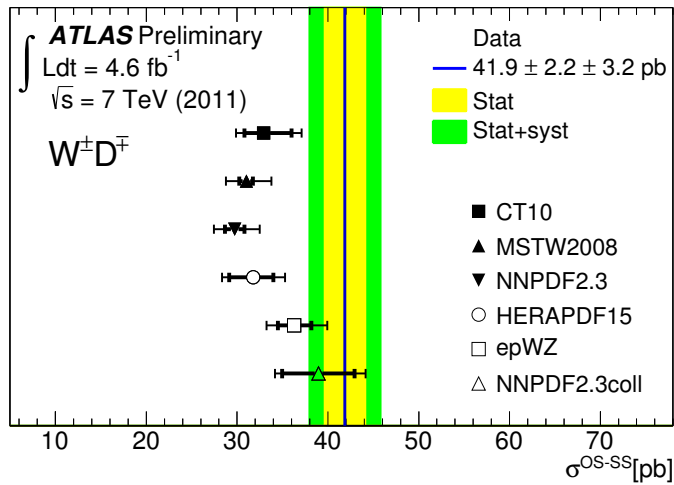

(b)

Figure 3: The measured $W^{ \pm}+D^{* \mp}$ (a) and $W^{ \pm}+D^{\mp}$ (b) OS-SS cross-sections, compared to six aMC@ NLO NLO predictions which each use a different PDF [18]. The aMC@ NLO predictions display PDF uncertainties evaluated using the error sets (inner error bars), and PDF plus scale uncertainties (outer error bars).

the strange quark density. The CT10, MSTW2008, NNPDF2.3 and HERAPDF15 PDFs all have an $s$ quark density that is suppressed versus the $d$ quark. The epWZ PDF has an $s$ quark and $d$ quark density that is comparable at $x \sim 0.01$, and the NNPDF2.3coll has a larger $s$ quark density than $d$ at all $x$. The results seem to favour PDFs where the $s$ quark and $d$ quark sea contributions are comparable at $x \sim 0.01$.

A measurement is also made of the differential OS-SS cross-section for $W^{ \pm}+D^{* \mp}$ and $W^{ \pm}+$ $D^{\mp}$ production as a function of the charm hadron $p_{T}\left(p_{T}^{D}\right)$ and the $\eta$ of the $W$ decay lepton. In this measurement the cross-section is normalised by the measured inclusive $W$ production rate. The measurement as a function of $p_{T}^{D}$ is shown in Figure 4, for two example $W^{ \pm}$and $D^{(*) \mp}$ final states. Reasonable agreement between the measurement and aMC@NLO with CT10 is observed, although there is an indication that the $p_{T}^{D}$ spectra of the prediction is perhaps too soft.

\section{Conclusions}

The excellent performance of the ATLAS tracking detectors and calorimetry, as well as the large amount of data delivered by the LHC in the 2011 running, have enabled the vector boson plus heavy flavour process to be probed with a new level of precision and scope, in particular the first differential cross-section measurements. This has yielded some interesting comparisons to the latest NLO predictions, showing potential limitations of the theory in some areas. In addition, the prospects for an improved understanding of the strange quark content of the proton through a study of the $W+c$ final state have been clearly demonstrated.

\section{References}

[1] ATLAS Collaboration, Phys. Rev. D 85 (2012) 092002.

[2] ATLAS Collaboration, JHEP 1307 (2013) 032. 


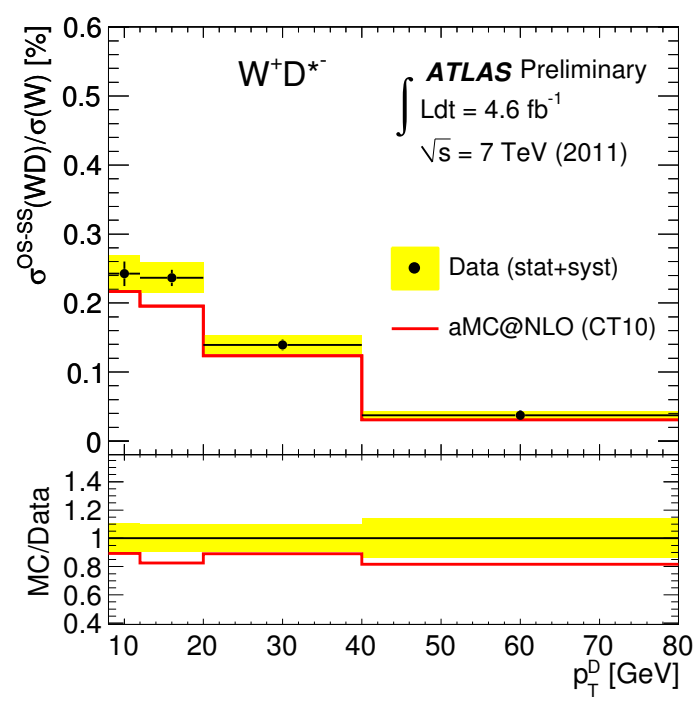

(a)

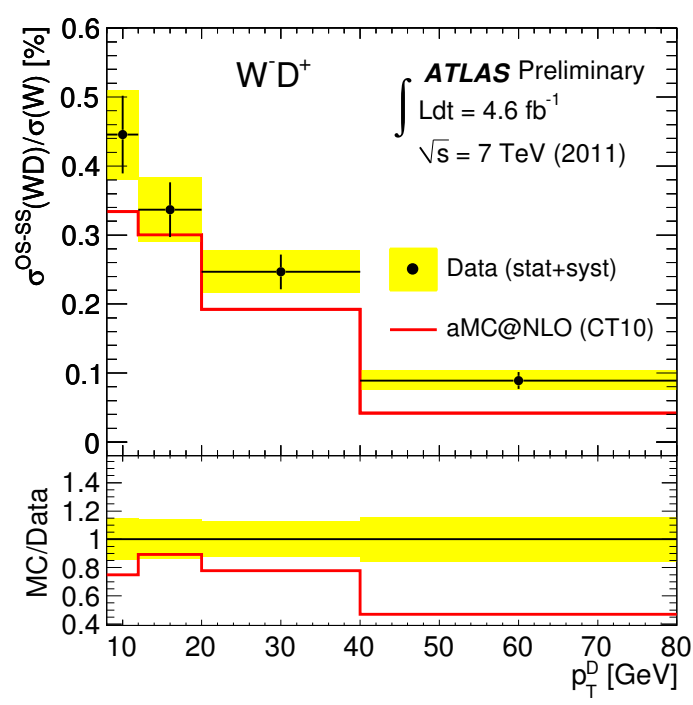

(b)

Figure 4: The measured differential $\sigma\left(W^{+}+D^{*-}\right) / \sigma\left(W^{+}\right)$(a) and $\sigma\left(W^{-}+D^{+}\right) / \sigma\left(W^{-}\right)$(b) OS-SS crosssections as a function of $p_{T}^{D}$, compared to aMC@NLO NLO predictions using the CT10 PDF [18].

[3] CMS Collaboration, JHEP 1201 (2012) 010.

[4] ATLAS Collaboration, Phys. Lett. B 720 (2013) 13.

[5] ATLAS Collaboration, ATLAS-CONF-2013-079, https://cds.cern.ch/record/1563235.

[6] CMS Collaboration, CMS-PAS-HIG-13-012, https://cds.cern.ch/record/1546801.

[7] ATLAS Collaboration, 2008 JINST 3 S08003.

[8] ATLAS Collaboration, Phys. Lett. B 706 (2012) 295.

[9] M. Cacciari, G. P. Salam, and G. Soyez, JHEP 0804 (2008) 063.

[10] J. M. Campbell, F. Caola, F. Febres Cordero, L. Reina and D. Wackeroth, Phys. Rev. D 86 (2012) 034021.

[11] M. L. Mangano, M. Moretti, F. Piccinini, R. Pittau and A. D. Polosa, JHEP 0307 (2003) 001.

[12] G. Corcella, I. G. Knowles, G. Marchesini, S. Moretti, K. Odagiri, P. Richardson, M. H. Seymour and B. R. Webber, JHEP 0101 (2001) 010.

[13] T. Gleisberg, S. .Hoeche, F. Krauss, M. Schonherr, S. Schumann, F. Siegert and J. Winter, JHEP 0902 (2009) 007.

[14] ATLAS Collaboration, JHEP 1306 (2013) 084.

[15] ATLAS Collaboration, ATLAS-CONF-2011-102, https://cds.cern.ch/record/1369219.

[16] S. Alioli, P. Nason, C. Oleari and E. Re, JHEP 1006 (2010) 043.

[17] T. Sjostrand, S. Mrenna and P. Z. Skands, JHEP 0605 (2006) 026.

[18] ATLAS Collaboration, ATLAS-CONF-2013-045, https://cds.cern.ch/record/1546800.

[19] R. Frederix, S. Frixione, V. Hirschi, F. Maltoni, R. Pittau and P. Torrielli, Phys. Lett. B 701 (2011) 427. 